\title{
Design and Validation of Real-Time Optimal Control with ECMS to Minimize Energy Consumption for Parallel Hybrid Electric Vehicles
}

\author{
Aiyun Gao, ${ }^{1}$ Xiaozhong Deng, ${ }^{2}$ Mingzhu Zhang, ${ }^{3}$ and Zhumu Fu ${ }^{4}$ \\ ${ }^{1}$ School of Mechatronics, Northwestern Polytechnical University, Xian 710072, China \\ ${ }^{2}$ Henan Collaborative Innovation Center of Machinery Equipment Advanced Manufacturing, \\ Henan University of Science and Technology, Luoyang 471003, China \\ ${ }^{3}$ School of Mechatronics Engineering, Henan University of Science and Technology, Luoyang 471003, China \\ ${ }^{4}$ School of Information Engineering, Henan University of Science and Technology, Luoyang 471023, China \\ Correspondence should be addressed to Xiaozhong Deng; eaglecloud@haust.edu.cn
}

Received 27 September 2016; Accepted 13 December 2016; Published 26 January 2017

Academic Editor: Michele Betti

Copyright (C) 2017 Aiyun Gao et al. This is an open access article distributed under the Creative Commons Attribution License, which permits unrestricted use, distribution, and reproduction in any medium, provided the original work is properly cited.

\begin{abstract}
A real-time optimal control of parallel hybrid electric vehicles (PHEVs) with the equivalent consumption minimization strategy (ECMS) is presented in this paper, whose purpose is to achieve the total equivalent fuel consumption minimization and to maintain the battery state of charge (SOC) within its operation range at all times simultaneously. Vehicle and assembly models of PHEVs are established, which provide the foundation for the following calculations. The ECMS is described in detail, in which an instantaneous cost function including the fuel energy and the electrical energy is proposed, whose emphasis is the computation of the equivalent factor. The real-time optimal control strategy is designed through regarding the minimum of the total equivalent fuel consumption as the control objective and the torque split factor as the control variable. The validation of the control strategy proposed is demonstrated both in the MATLAB/Simulink/Advisor environment and under actual transportation conditions by comparing the fuel economy, the charge sustainability, and parts performance with other three control strategies under different driving cycles including standard, actual, and real-time road conditions. Through numerical simulations and real vehicle tests, the accuracy of the approach used for the evaluation of the equivalent factor is confirmed, and the potential of the proposed control strategy in terms of fuel economy and keeping the deviations of SOC at a low level is illustrated.
\end{abstract}

\section{Introduction}

Hybrid electric vehicles (HEVs) are most promising among all the new energy vehicles including battery electric vehicles and fuel cell vehicles to better fuel economy and emissions without compromising vehicle performances $[1,2]$. In the last few decades, many automobile manufacturers have been researching HEVs and have obtained several configurations for practical applications $[3,4]$. There are many methods to improve fuel economy of HEVs, such as optimizing their mechanical construction, matching the powertrain parameters, and lighting the body. This paper will optimize the energy management strategy, which distributes the total torque demanded at wheels between the ICE and the electric motor (EM) to minimize the fuel consumption and maintain the battery state of charge (SOC) simultaneously.

Many energy management strategies have been proposed for efficient energy usage, which can be classified into four types, namely, the rule-based control strategy, the global optimal strategy, the real-time optimization control strategy, and the fuzzy logic control strategy. The rule-based control strategy sets the initial value of the parameters by mostly relying on engineering experience and then adjusts these parameters by adopting the trial-and-error method. Although this strategy can offer a prominent improvement in energy efficiency and is also adopted widely in the commercial HEV, it is clear that the strategy does not guarantee an optimal value in all cases or allow the vehicle to run at maximum efficiency 
when the parameters are fixed $[5,6]$. Although the fuzzy logic control strategy is good at dealing with model uncertainty and complex decisions, the formulation of its fuzzy rules is lack of system approach and mainly depends on engineering experience, which leads to loss of control accuracy $[7,8]$.

The papers $[9,10]$ propose a global optimal strategy based on dynamic programming methods for parallel hybrid electric vehicles (PHEVs) and parallel-series HEV, respectively. These techniques can find a global optimal solution to the control parameters, such as the ICE/EM torque, but cannot offer an online solution because they assume that the future driving cycle is entirely known. This method can be a good analysis and assessment tool for other control strategies. However, due to computational complexity, it is not easily implemented for practical applications.

Due to the causal nature of global optimization technique, it is not suitable for real-time analysis, because the main aim of the real-time analysis is to reduce global criterion to an instantaneous optimization by introducing a cost function that depends only on the present state of the system parameters $[11,12]$. Moreover, global optimization technique does not consider variations of battery SOC in the problem. Hence, in order to derive cost functions for instantaneous optimization of power split, while maintaining battery SOC, real-time optimization is performed.

The real-time control strategy is based on instantaneous optimization and defines a cost function which is guaranteed to be minimum at each instant depending upon system current variables. Various attempts have been made to propose real-time control based on instantaneous optimization [1315]. The more promising approach of the real-time optimization is used in [16], which is defined as equivalent consumption minimization strategy (ECMS). Its cost function is a sum of the ICE fuel consumption and the EM equivalent fuel consumption. To be distinguished, the real-time control strategy without ECMS is defined as the simpler real-time strategy.

The ECMS is supposing that the energy consumption from the battery at present is supplemented by the ICE in the future. Thus, battery discharging at any time is equivalent to fuel consumption of the ICE in the future. Due to the small computational time, near-optimal characteristics, and the feasibility of online implementation, ECMS has widely been used to address the energy management control problem for both HEVs $[17,18]$ and PHEVs $[19,20]$. The key problem of the ECMS design is the calculation of the equivalent factor between fuel and electrical energy based on the available vehicle information, because it has a major influence on the fuel economy and the charge sustainability of PHEVs.

In this paper, a real-time control strategy with ECMS for a PHEV is proposed, which is based on a new method for evaluating the equivalent factor between fuel and electrical energy in order to regulate SOC at a constant reference point with the minimum fuel consumption simultaneously. Based on the models established of the PHEV, computation and optimization of the total equivalent fuel consumption are discussed in detail in the paper.

The remainder of this paper is organized as follows. Section 2 introduces the vehicle configuration and models of the parallel hybrid electric vehicle. Section 3 then describes

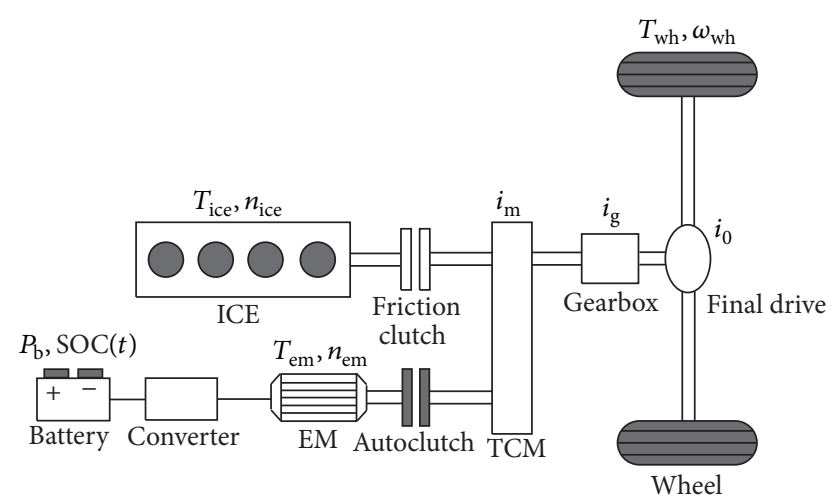

Figure 1: Schematic diagram of the PHEV.

the novel ECMS algorithm. The design of the real-time optimal control is presented in Section 4. Validation of the control strategy proposed and optimization results are discussed in Section 5. Section 6 highlights this paper's key conclusions.

\section{Configuration and Models of the PHEV}

2.1. Configuration of the PHEV. The principal schematic of the PHEV is shown in Figure 1. Both the output torque of the ICE and the EM are coupled by the torque coupling mechanism (TCM), whose output torque is then transmitted into the gearbox and final drive, through which the vehicle is ultimately propelled. A friction clutch is located between the ICE and the TCM, and an autoclutch is located between the EM and the TCM. The main component specifications of the hybrid powertrain system are listed in Table 1.

\subsection{Models of the PHEV}

2.2.1. Vehicle Dynamics Models. Based on the wheel force balance, vehicle dynamics models must give power balance equation in every time step of simulation computation. Therefore, they are composed of dynamics equation and power balance equation. Dynamics equation is described by the following:

$$
\begin{aligned}
F_{t}= & F_{f}+F_{i}+F_{w}+F_{j}, \\
\text { or } & \frac{\left(T_{\mathrm{ice}}+T_{\mathrm{em}} \cdot i_{m}\right) i_{\mathrm{g}} i_{0} \eta_{T}}{r} \\
= & m g f\left(u_{a}(t)\right) \cos \alpha(t)+m g \sin \alpha(t) \\
& \quad+\frac{C_{D} A u_{a}^{2}(t)}{21.15}+\delta m \frac{\mathrm{d} u_{a}(t)}{\mathrm{d} t},
\end{aligned}
$$

where $F_{t}$ is the driving force, $F_{f}$ is the rolling resistance, $F_{i}$ is the gradient resistance, $F_{w}$ is the air resistance, $F_{j}$ is the acceleration resistance, $T_{\text {ice }}$ is the ICE torque, $T_{\mathrm{em}}$ is the EM torque, $i_{m}$ is the TCM ratio, $i_{\mathrm{g}}$ is the gearbox ratio, $i_{0}$ is the final drive ratio, $\eta_{T}$ is the powertrain efficiency, $r$ is the wheel rolling radius, $m$ is the vehicle mass, $g$ is the acceleration of gravity, $f\left(u_{a}(t)\right)$ is the rolling resistance coefficient, $\alpha$ is 
TABLE 1: Numerical values of the vehicle parameters.

\begin{tabular}{|c|c|c|}
\hline Component & Parameter & Value \\
\hline \multirow{7}{*}{ Vehicle } & $\begin{array}{l}\text { Air resistance } \\
\text { coefficient } C_{D}\end{array}$ & 0.31 \\
\hline & Frontal area $A$ & $2 \mathrm{~m}^{2}$ \\
\hline & $\begin{array}{l}\text { Vehicle total mass } \\
m\end{array}$ & $2081 \mathrm{~kg}$ \\
\hline & $\begin{array}{l}\text { Wheel rolling } \\
\text { radius } r\end{array}$ & $0.283 \mathrm{~m}$ \\
\hline & $\begin{array}{l}\text { Rolling resistance } \\
\text { coefficients } f_{0}\end{array}$ & $1.1 \times 10^{-2}$ \\
\hline & $\begin{array}{l}\text { Rolling resistance } \\
\text { coefficients } f_{1}\end{array}$ & $2.6 \times 10^{-4}$ \\
\hline & $\begin{array}{l}\text { Rolling resistance } \\
\text { coefficients } f_{4}\end{array}$ & $6.5 \times 10^{-4}$ \\
\hline \multirow{3}{*}{ ICE } & $\begin{array}{l}\text { Maximum power } \\
P_{\text {ice } \max }\end{array}$ & $30 \mathrm{kw}(3500 \mathrm{r} / \mathrm{min})$ \\
\hline & $\begin{array}{l}\text { Maximum torque } \\
T_{\text {ice } \max }\end{array}$ & $81 \mathrm{Nm}(3500 \mathrm{r} / \mathrm{min})$ \\
\hline & Peak efficiency & 0.39 \\
\hline \multirow{3}{*}{ EM } & $\begin{array}{l}\text { Maximum power } \\
P_{\mathrm{em} \max }\end{array}$ & $25 \mathrm{kw}$ \\
\hline & $\begin{array}{l}\text { Maximum torque } \\
T_{\text {em } \max }\end{array}$ & $160 \mathrm{Nm}$ \\
\hline & Peak efficiency & 0.90 \\
\hline \multirow{4}{*}{ Powertrain } & $\begin{array}{l}\text { Drivetrain } \\
\text { efficiency } \eta_{T}\end{array}$ & 0.9 \\
\hline & Gearbox ratio $i_{\mathrm{g}}$ & {$[3.78,2.12,1.35,0.97,0.76]$} \\
\hline & Final drive ratio $i_{0}$ & 3.24 \\
\hline & TCM ratio $i_{m}$ & 1.3 \\
\hline
\end{tabular}

the gradient angle, $C_{D}$ is the air resistance coefficient, $A$ is the frontal area, $u_{a}(t)$ is the vehicle velocity, and $\delta$ is the conversion coefficient of inertia mass.

Under good road conditions, the rolling resistance coefficient $f\left(u_{a}(t)\right)$ of the vehicle is estimated as a fourth-order polynomial function of the vehicle velocity $u_{a}(t)$ [21]

$$
f\left(u_{a}(t)\right)=f_{0}+f_{1}\left(\frac{u_{a}(t)}{100}\right)+f_{4}\left(\frac{u_{a}(t)}{100}\right)^{4},
$$

where $f_{0}, f_{1}$, and $f_{4}$ are coefficients, obtained from vehicle theory.

Power balance equation is shown as

$$
\begin{aligned}
P_{\text {ice }} & +P_{\text {em }}=\frac{1}{3600 \eta_{T}}\left(m g f\left(u_{a}(t)\right) u_{a}(t)+m g i u_{a}(t)\right. \\
+ & \left.\frac{C_{D} A\left(u_{a}(t)\right)^{3}}{21.15}+\delta m u_{a}(t) \frac{\mathrm{d} u_{a}(t)}{\mathrm{d} t}\right),
\end{aligned}
$$

where $P_{\text {ice }}$ is the ICE power, $P_{\mathrm{em}}$ is the EM power, $i$ is gradability, and the others are the same as in (2).

2.2.2. ICE Models. The ICE numerical model is established based on the steady-state test data of the ICE. By bench test,

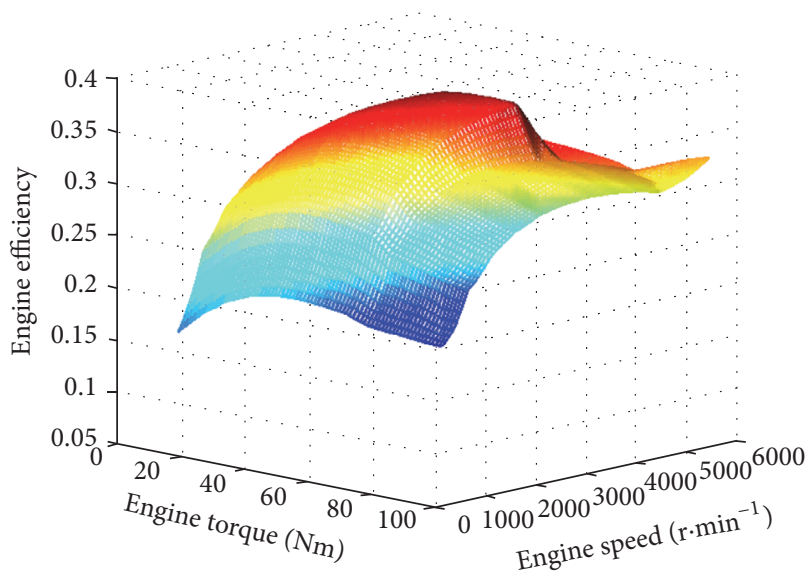

FIGURE 2: Steady operating efficiency map of the ICE.

the efficiency map of the ICE in three-dimensional space of efficiency-speed-torque is obtained and shown in Figure 2.

The ICE fuel consumption model is as below:

$$
Q_{\text {ice }}(t)=f_{\text {ice }}\left(T_{\text {ice }}(t), n_{\text {ice }}(t)\right)=\frac{P_{\text {ice }} b_{\text {ice }}(t)}{1.02 u_{a}(t) \cdot \rho \cdot g},
$$

where $Q_{\text {ice }}(t)$ is the ICE fuel consumption per $100 \mathrm{~km}, n_{\text {ice }}(t)$ is the ICE speed, $b_{\text {ice }}(t)$ is the ICE fuel consumption rate, and $\rho$ is the fuel density.

The function $f_{\text {ice }}$ can be obtained from the ICE bench tests. Using the ICE mapping characteristics curve, $b_{\text {ice }}(t)$ is calculated as follows:

$$
\begin{aligned}
& b_{\mathrm{ice}}(t) \\
& \quad=\sum_{j=0}^{s} \sum_{i=0}^{j} B\left[\frac{1}{2}(j+1)(j+2)-j-1+i\right] \cdot T_{\mathrm{ice}}^{i} \cdot n_{\mathrm{ice}}^{j-1},
\end{aligned}
$$

where $s$ is the model order and $B$ is the model coefficient group.

2.2.3. EM Model. A multiphase Permanent Magnet Synchronous Machine with a direct torque controller is used as the EM of the PHEV. Since the response time of the EM is in milliseconds and much faster than the mechanical time of the vehicle and its powertrain, the EM transient response could be neglected. Similar to the ICE, the efficiency maps of the EM are achieved by fitting. The EM characteristics are based on the efficiency data obtained from experiments. Figure 3 shows the output torque-speed characteristics and efficiency map of the EM system. The EM efficiency is a function of the torque $T_{\mathrm{em}}$ and speed $n_{\mathrm{em}}$ of the EM: that is, $\eta_{\mathrm{em}}=f\left(T_{\mathrm{em}}, n_{\mathrm{em}}\right)$.

2.2.4. Gearbox Model. The dynamics relationships among the ICE, EM, and the gearbox torques are given by the following equation:

$$
T_{\text {ice }}(t)+T_{\mathrm{em}}(t) \cdot i_{m}=T_{\mathrm{g}},
$$

where $T_{\mathrm{g}}(t)$ is the gearbox torque and the values of $T_{\mathrm{em}}(t)$ and $T_{\text {ice }}(t)$ are determined by the optimization results of the realtime optimal control in Section 4. 


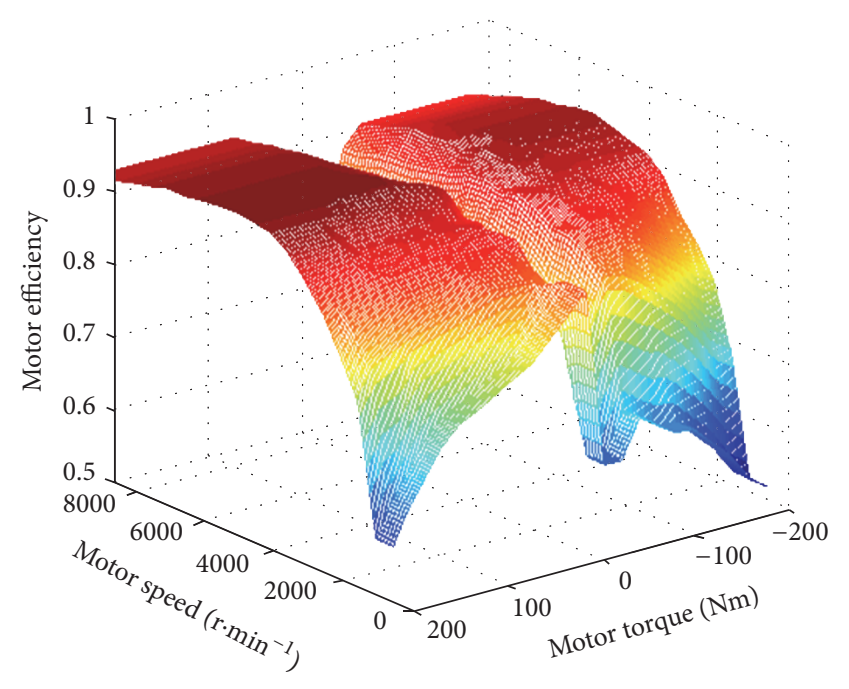

FIGURE 3: Steady operating efficiency map of the EM.

2.2.5. Battery Models. NIMH batteries are selected as energy storage mechanism. The battery model is simplified as the circuit system in which an ideal voltage source is in series with an equivalent internal resistance. The battery SOC can be predicted by the following equation:

$$
\begin{aligned}
\mathrm{SOC} & =\mathrm{SOC}_{0}-\frac{\int_{T_{0}}^{T} i_{\mathrm{b}} \mathrm{d} t}{C_{\mathrm{b}}} \\
& =\mathrm{SOC}_{0}-\frac{\int_{T_{0}}^{T} U_{\mathrm{oc}}-\left(U_{\mathrm{oc}}{ }^{2}-4 R_{\mathrm{b}} P_{\mathrm{b}}\right)^{1 / 2} \mathrm{~d} t}{2 R_{\mathrm{b}} C_{\mathrm{b}}},
\end{aligned}
$$

where $\mathrm{SOC}_{0}$ is the initial value of the battery SOC, $T_{0}$ is the initial value of the time, $i_{\mathrm{b}}$ is the charging or discharging current, $C_{\mathrm{b}}$ is the total capacity, $U_{\mathrm{oc}}$ is the open circuit voltage, $R_{\mathrm{b}}$ is the equivalent internal resistance, and $P_{\mathrm{b}}$ is the effective power of the battery.

The battery SOC is the main dynamic state in optimal control of a PHEV and its dynamics can be described as follows:

$$
\frac{\mathrm{dSOC}}{\mathrm{d} t}=-\frac{U_{\mathrm{oc}}-\sqrt{U_{\mathrm{oc}}^{2}(\mathrm{SOC})-4 R_{\mathrm{b}}\left(\mathrm{SOC}, i_{\mathrm{b}}\right) P_{\mathrm{b}}}}{2 Q_{\max } R_{\mathrm{b}}\left(\mathrm{SOC}, i_{\mathrm{b}}\right)},
$$

where $\mathrm{dSOC} / \mathrm{d} t$ is the battery SOC rate and $Q_{\max }$ is the battery charge capacity.

By combining (8) and (9), the final control-oriented battery models can be obtained. The calculation model of $R_{\mathrm{b}}$ and $U_{\mathrm{oc}}$ and the estimation model of the SOC are shown in Figure 4 .

2.2.6. Wheels Models. The vehicle demanding torque at the wheels $T_{\mathrm{dem}}(t)$ is calculated as follows:

$$
\begin{aligned}
T_{\text {dem }}(t) & =\left(T_{\text {ice }}(t)+T_{\text {em }}(t) \cdot i_{m}\right) \cdot i_{\mathrm{g}} \cdot i_{0} \\
& =T_{\text {ice }}(t) \cdot i_{\mathrm{g}} \cdot i_{0}+T_{\text {em }}(t) \cdot i_{m} \cdot i_{\mathrm{g}} \cdot i_{0} \\
& =T_{\mathrm{f} \text { path }}(t)+T_{\text {e path }}(t),
\end{aligned}
$$

where $T_{\mathrm{f} \text { path }}(t)$ is the torque provided by the fuel path and $T_{\text {e path }}(t)$ is the torque provided by the electrical path.

The control variable is the torque split factor that manages the torque distribution between the electrical path and the fuel path. Its definition is as below:

$$
k(t)=\frac{T_{\text {e path }}}{T_{\text {dem }}} .
$$

Therefore, when the value $k(t)=1$, all the positive torque demanded at the wheels is supplied by the electrical path. When $k(t)=0$, all the torque needed at the wheels is offered by the fuel path.

The driving wheel torque $T_{\mathrm{wh}}(t)$ and angular speed $\omega_{\mathrm{wh}}(t)$ are described as follows:

$$
\begin{aligned}
T_{\mathrm{dem}}(t) & =T_{\mathrm{wh}}(t)=r \cdot F_{t}(t), \\
\omega_{\mathrm{wh}}(t) & =\frac{u_{a}(t)}{r} .
\end{aligned}
$$

2.2.7. Energy Models. The ICE energy $E_{\text {ice }}(t)$, the EM energy $E_{\mathrm{em}}(t)$, and the vehicle demanding energy $E_{\mathrm{dem}}(t)$ are the following:

$$
\begin{gathered}
E_{\text {ice }}(t)=\int_{0}^{t} \frac{Q_{\text {ice }}(\tau) \cdot 1.02 u_{a}(\tau) \rho g}{b_{\text {ice }}(\tau)} \mathrm{d} \tau, \\
E_{\text {em }}(t)=\int_{0}^{t} i_{\mathrm{b}}(\tau) U_{\mathrm{oc}}(\tau) d \tau, \\
E_{\mathrm{dem}}(t)=E_{\mathrm{wh}}(t)=\int_{0}^{t} T_{\mathrm{wh}}(\tau) \omega_{\mathrm{wh}}(\tau) d \tau .
\end{gathered}
$$

\section{Novel ECMS Algorithm}

The total equivalent fuel consumption is regarded as the control objective for optimization and the torque split factor $k(t)$ as the control variable of the ECMS. The total vehicle equivalent fuel consumption $J$ is

$$
J(t, k)=\int_{0}^{T}\left(\frac{\mathrm{d} m_{\text {ice }}(k(t), t)}{\mathrm{d} t}+k_{\mathrm{eq}} \frac{\mathrm{d} m_{\mathrm{em}}(t)}{\mathrm{d} t}\right) \mathrm{d} t,
$$

where 0 and $T$ are, respectively, the initial and the final times of the given driving cycle, $\mathrm{d} m_{\text {ice }} / \mathrm{d} t$ is the fuel consumption per unit time of the ICE, $k_{\text {eq }}$ is the equivalent factor that acts as a weighting factor for the electrical energy, and $\mathrm{d} m_{\mathrm{em}} / \mathrm{d} t$ is the electrical consumption per unit time of the EM during charging or discharging the battery. When the EM charges the battery,

$$
\frac{\mathrm{d} m_{\mathrm{em}}}{\mathrm{d} t}=\frac{P_{\mathrm{em}} \cdot \eta_{\mathrm{e}}}{\mathrm{Q}_{\mathrm{lhv}}},
$$

and when the EM discharges the battery,

$$
\frac{\mathrm{d} m_{\mathrm{em}}}{\mathrm{d} t}=\frac{P_{\mathrm{em}}}{\eta_{\mathrm{e}} \cdot Q_{\mathrm{lhv}}},
$$

where $\eta_{\mathrm{e}}$ is the efficiency of the electrical path and $Q_{\mathrm{lhv}}$ is the fuel lower heating value. The calculation of the equivalent 


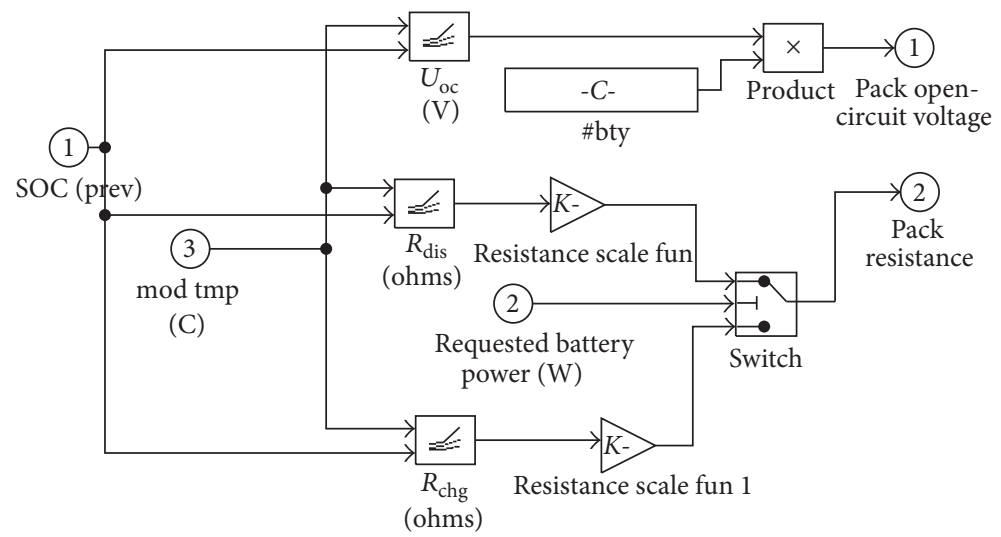

(a) Calculation model of $R_{\mathrm{b}}$ and $U_{\mathrm{oc}}$

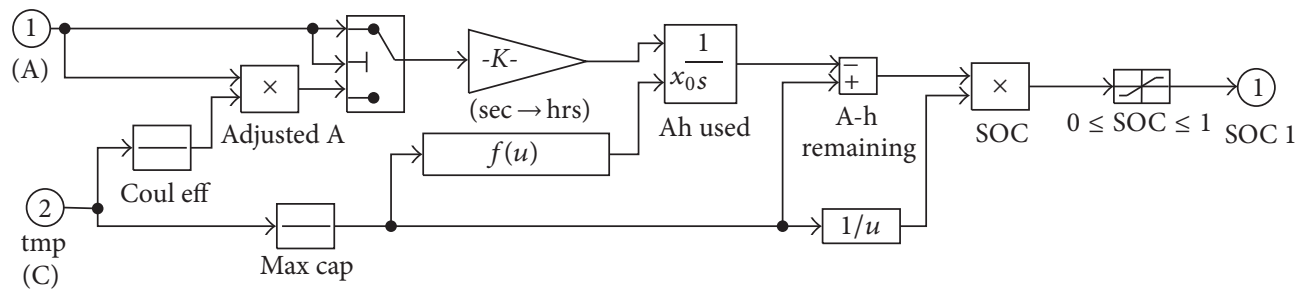

(b) Estimation model of the SOC

Figure 4: Battery models.

factor $k_{\mathrm{eq}}$ is the key problem of the ECMS design because it affects torque split factor $k(t)$ whose optimum would be different under different driving cycles.

Seen from (14), $J$ does not take into consideration the battery SOC explicitly, so it cannot keep the deviations of SOC at a low level. Therefore, a feedback correction is applied to $k_{\text {eq }}$ based on the SOC. The corrected $k_{\text {eq }}$ is computed as follows:

$$
k_{\mathrm{eq}}=k_{\mathrm{neq}} \cdot k_{\mathrm{g} 1} \cdot k_{\mathrm{g} 2} \text {, }
$$

where $k_{\text {neq }}$ is the nominal equivalent factor. $k_{\text {neq }}$ cannot be too large or too small to use the electrical energy reasonably. The value of $k_{\text {neq }}$ can be estimated by simulations and it is different in accordance with the different driving circle. $k_{\mathrm{g} 1}$ and $k_{\mathrm{g} 2}$ gains are reckoned as below as a result of simulations conclusions over a long period of time:

$$
k_{\mathrm{g} 1}=1-\frac{\operatorname{SOC}(t)-\mathrm{SOC}_{\mathrm{ref}}}{1000 \times \Delta \mathrm{SOC}}
$$

$$
k_{\mathrm{g} 2}=1+\tan \left(10 \times\left(\operatorname{SOC}(t)-\mathrm{SOC}_{\mathrm{ref}}\right)\right) \text {, }
$$

where $\mathrm{SOC}_{\text {ref }}$ is the reference SOC of the ECMS algorithm and $\triangle \mathrm{SOC}$ is the allowable range of SOC around $\mathrm{SOC}_{\text {ref }}$.

From (18), the value and deviation of the battery SOC are converted into equivalent fuel consumption. This makes the method evaluate the actual fuel economy of PHEV by taking into account SOC deviation as well.

\section{Design of Real-Time Optimal Control with ECMS}

4.1. Problem Formulation. The optimal control objective of the PHEV minimizes the total vehicle equivalent fuel consumption $J$, which is global over the whole driving cycle. However, the control action of the PHEV is local. In addition, it is necessary to satisfy some constrains, such as the battery SOC, the main components limitations to ensure their safe operation. In a word, the problem formulation is as below:

$$
\min \left\{J(t, k)=\int_{0}^{T}\left(\frac{\mathrm{d} m_{\mathrm{ice}}(k(t), t)}{\mathrm{d} t}+k_{\mathrm{eq}} \frac{\mathrm{d} m_{\mathrm{em}}(t)}{\mathrm{d} t}\right) \mathrm{d} t\right\}
$$

subject to: $\quad 0 \leq T_{\text {ice }}(t) \leq T_{\text {ice_max }}\left(n_{\text {ice }}(t)\right)$

$$
\begin{aligned}
& n_{\text {ice_min }}(t) \leq n_{\text {ice }}(t) \leq n_{\text {ice_max }}(t) \\
& T_{\text {em_min }}\left(n_{\text {em }}(t)\right) \leq T_{\text {em }}(t) \leq T_{\text {em_max }}\left(n_{\text {em }}(t)\right)
\end{aligned}
$$




$$
\begin{aligned}
& n_{\text {em_min }}(t) \leq n_{\mathrm{em}}(t) \leq n_{\mathrm{em} \_ \text {max }}(t) \\
& \operatorname{SOC}_{\text {min }}(t) \leq \mathrm{SOC}(t) \leq \operatorname{SOC}_{\text {max }}(t) \\
& \operatorname{SOCC}=f(k(t), t), \\
& \operatorname{SOC}(0)=\operatorname{SOC}_{0} \\
& \forall t,
\end{aligned}
$$

where _ min and _ max denote the minimum and maximum bounds which are generally variable and $\operatorname{SOC}_{\text {min }}(t)$ and $\mathrm{SOC}_{\max }(t)$ are the minimum and maximum SOC values according to the battery's physical constraints on the assumption that $\mathrm{SOC}(0)$ equals $\mathrm{SOC}_{0}$.

According to this, the state variable $x$, control inputs $u$, and control outputs $o$ of the real-time optimal controller models are as below, respectively:

$$
\begin{aligned}
& x=\operatorname{SOC}(t), \\
& u=\left[\begin{array}{c}
T_{\text {dem }} \\
n_{\text {ice }} \\
k(t)
\end{array}\right], \\
& o=\left[\begin{array}{c}
T_{\text {ice,opt }}(t) \\
T_{\text {em }, \text { opt }}(t) \\
J(t, k)
\end{array}\right] .
\end{aligned}
$$

4.2. Information of the Driving Cycle. The information of the driving cycle can be obtained through the global positioning system (GPS), the intelligent transportation system (ITS), and on-board sensors like Distronic, which is a radar system for detecting the distance to the car ahead. As shown in Figure 5, the historical traffic information is transferred to the predictive models of the driving cycle; then the uncorrected nominal equivalent factor $k_{\text {neq }}^{\prime}$ is obtained in accordance with the different driving cycle. The uncertainty of instantaneous traffic flow can be regarded as a particular statistical distribution and can be considered to be stochastic disturbances in the trip model. Based on the real-time information of the driving cycle, $k_{\text {neq }}^{\prime}$ is revised and the nominal equivalent factor $k_{\text {neq }}$ is acquired. At the same time, $\mathrm{SOC}_{\text {ref }}$ generates a time-varying trajectory to avoid the events in which the battery's SOC runs into its constraints due to recuperation. Finally, the instantaneous $k_{\text {eq }}$ is input into the real-time optimal control and can be used to calculate the equivalent fuel consumption. This makes the method evaluate the actual fuel economy of PHEV by taking into consideration the driving cycle as well.

4.3. Real-Time Optimal Control Strategy with ECMS. The proposed real-time optimal control strategy with the ECMS is shown in Figure 6. ECMS computes the torque split factor to minimize a cost function at every moment. This control strategy is computationally cheap and has good robustness. Furthermore, its result is suboptimal next to that of the global optimization. Therefore, the match of the equivalent cost with the current driving cycle is critical and can be achieved by a device. The device is general GPS, ITS, and so on, mentioned in Section 4.2, through which the external information about the elevation and velocity profile of the driving cycle is transmitted to the driver model. The driver model decides the brake pedal, accelerator position, and clutch state based on the current vehicle $T_{\mathrm{dem}}(t), u_{a}$, and the driving cycle. The ECMS calculates the operating points of the ICE and the EM, which minimizes the equivalent fuel consumption and satisfies constraints such as the driver's power demand, speed limits, and torque limits. The objective function of the ECMS is represented in (14), where the design variable is selected as the torque split factor $k(t)$. The equivalent factor $k_{\text {eq }}$ has taken account of SOC deviation seen in Section 3, whose calculations in detail are as the following flow of the optimization. The ECMS can be the criterion to determine the operating points and to change the vehicle operating mode between the electrical vehicle (EV) and HEV modes. At last, $T_{\text {em,opt }}(t)$ and $T_{\text {ice,opt }}(t)$ are inputs to the EM and the ICE, respectively, to drive their working.

4.4. Flow of the Optimization. In order to make the optimization easy to understand, the flowchart of the optimization is shown in Figure 7. The optimization algorithm runs the following steps.

(1) Determine the possible working area using the following equation: $k=k_{1}: k_{\text {step }}: k_{\mathrm{h}}$, where $k$ is set in the range $k \in\left(k_{\mathrm{l}}, k_{\mathrm{h}}\right)$ given by the upper and lower bounds of the SOC that are admissible during the vehicle operation, $k_{\text {step }}$ is the calculation step of the torque split factor, and the smaller the selected $k_{\text {step }}$ is, the more precise the calculation result is.

(2) Compute $\mathrm{d} m_{\text {ice }} / \mathrm{d} t, \mathrm{~d} m_{\mathrm{em}} / \mathrm{d} t, T_{\text {wh }}, \omega_{\text {wh }}$ by using (2)(13), (15)-(16) and looking up the map of the ICE and the EM for each candidate operating point.

(3) Calculate the nominal equivalent factor $k_{\text {neq. }}$. Firstly, some driving cycles are obtained by statistic analysis based on a lot of measure data, depicted in Section 4.2. Secondly, under a certain driving cycle, the corresponding $k_{\text {neq }}^{\prime}$ is determined through many simulations. In the same way, $k_{\text {neq }}^{\prime}$ corresponding to other driving cycles is also received. Lastly, $k_{\text {neq }}^{\prime}$ needs be 


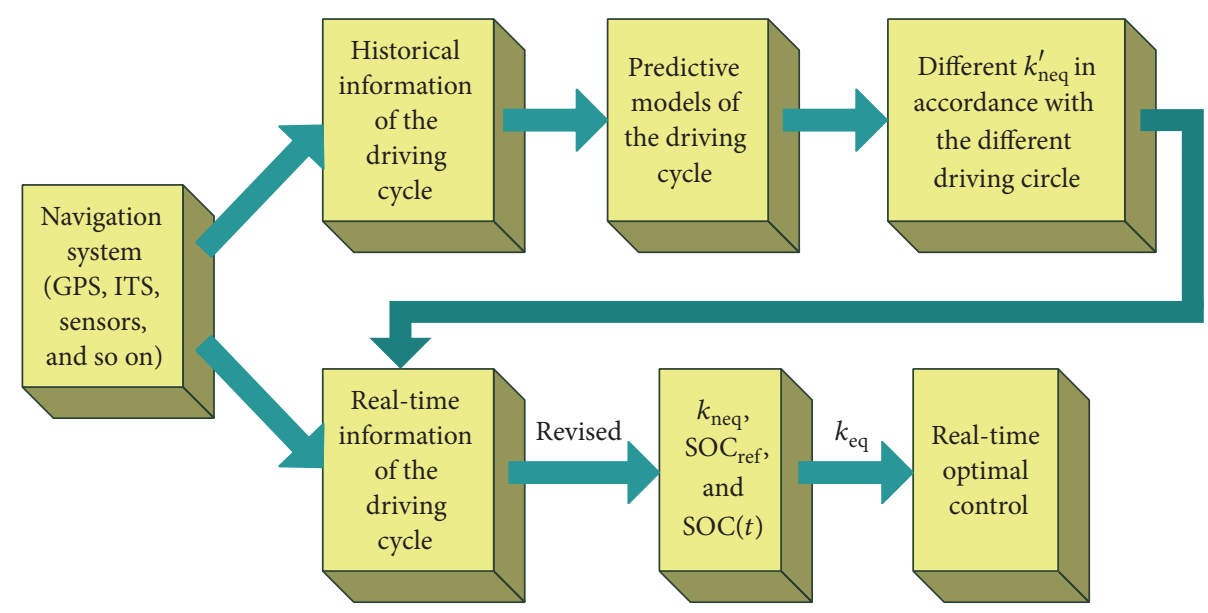

FIGURE 5: Information flowchart of the driving cycle.

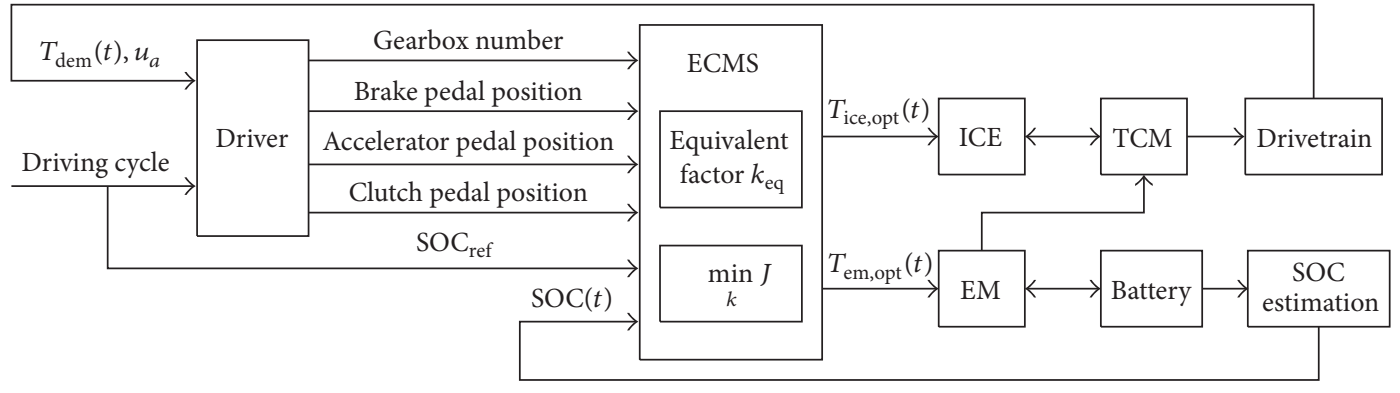

FIGURE 6: Real-time optimal control strategy with ECMS.

revised to make it more suitable for the real-time driving cycle. Then, the nominal equivalent factor $k_{\text {neq }}$ is acquired.

(4) $k_{\mathrm{g} 1}$ and $k_{\mathrm{g} 2}$ gains are computed by using (18), in which $\triangle \mathrm{SOC}$ is set according to the PHEV operating mode. $\mathrm{SOC}(t)$ is estimated by SOC estimation according to the real-time driving cycle and computed for each candidate operating point. $\mathrm{SOC}_{\text {ref }}$ is detected for each moment to ensure that SOC falls within the scope of $\left(\mathrm{SOC}_{\min }, \mathrm{SOC}_{\max }\right)$.

(5) The equivalence factor $k_{\text {eq }}$ is obtained by inputting $k_{\mathrm{neq}}, k_{\mathrm{g} 1}$, and $k_{\mathrm{g} 2}$ to (17).

(6) The equivalence factor $k_{\text {eq }}$ is input to the proposed real-time optimal control strategy with the ECMS; the total equivalent fuel consumption $J(t, k)$ is computed for each candidate operating point in MATLAB/Simulink/Advisor simulation software. The minimum value min $J(t, k)$ is selected in all $J(t, k)$ values and the corresponding index $i$ is recorded. The corresponding torque split factor $k$ is the optimal value $k_{\mathrm{opt}}(t)$ and the optimal torque split is achieved between the ICE and the EM.

\section{Simulation and Experiments Validation}

5.1. Simulation Validation. Numerical values of the vehicle parameters are shown in Table 1.

Firstly, the validation of the control strategy proposed is demonstrated in the MATLAB/Simulink/Advisor environment by comparing the fuel economy and charge sustainability with those offered by (1) the rule-based control strategy, (2) the global optimal control strategy, and (3) the simpler real-time control strategy. Their definitions, advantages, and disadvantages are described in Section 1.

The control performance considered in the following includes (1) the specific fuel consumption (SFC, liters/ $100 \mathrm{~km}$ ), (2) the equivalent specific fuel consumption (eSFC, liters $/ 100 \mathrm{~km}$ ), which is the sum of the fuel energy use and of the electrical energy use weighted by the equivalence factor $k_{\text {eq }}$, (3) the final battery SOC reached, starting from a value of 0.7 , and (4) the battery SOC variation with the time. The simulations are conducted on a set of standard driving profiles extracted from a database of fleet study data to statistically represent typical usage conditions of a PHEV.

Based on the use of the equivalent factors evaluated for the current driving cycle, the simulation results are shown in 


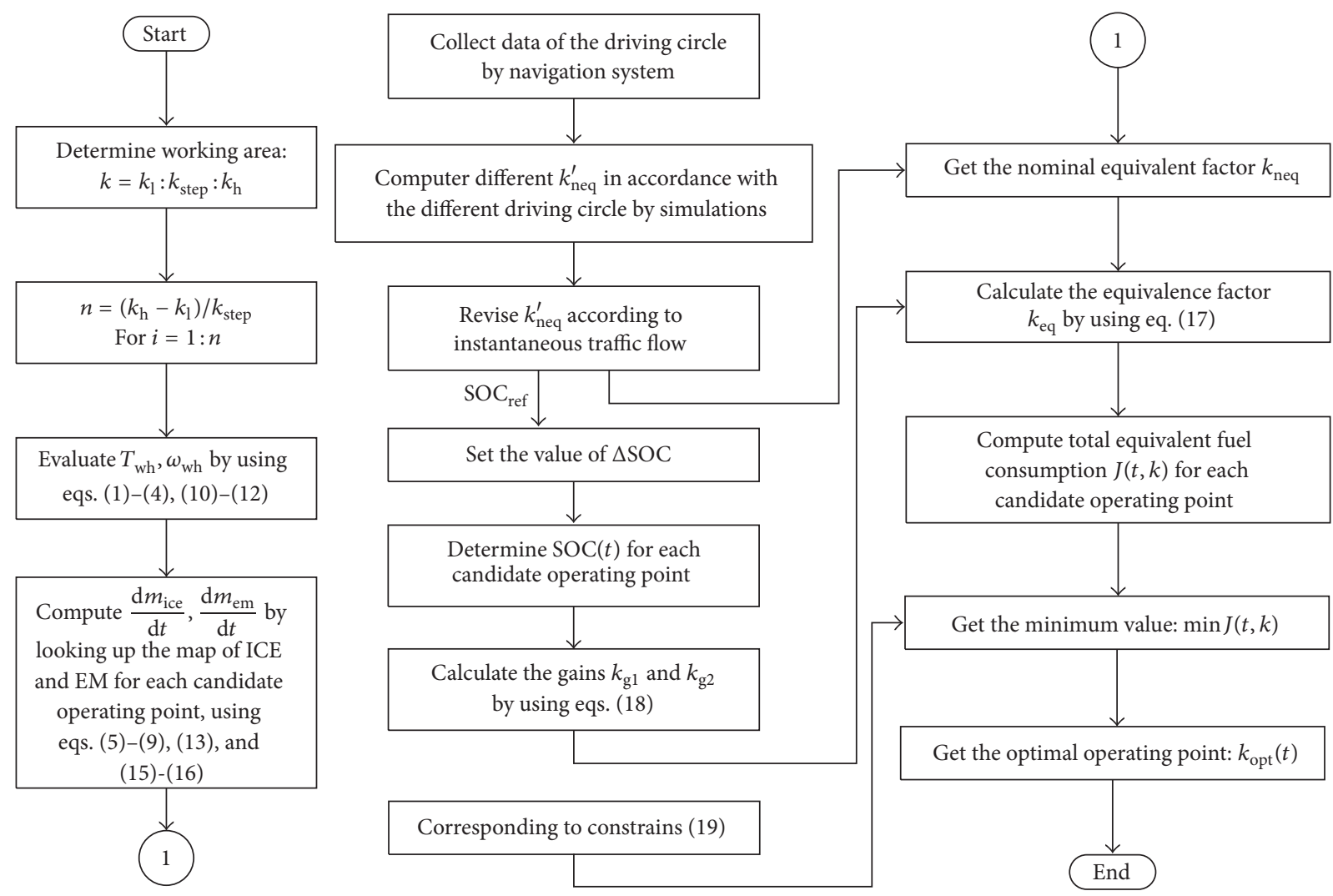

FIgURE 7: Flowchart of the optimization algorithm.

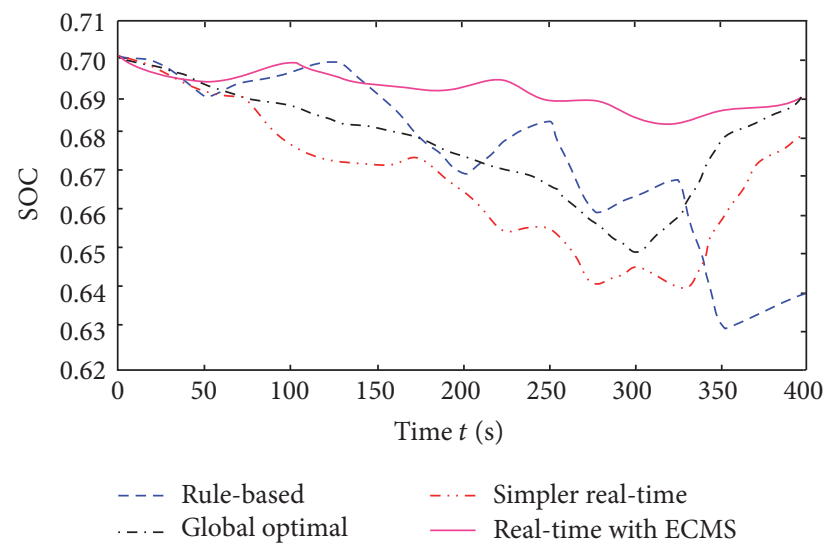

FIGURE 8: Battery SOC variation comparison of four control strategies with the time for a cycle under the EUDC.

Table 2 and Figure 8 under the Extra Urban Driving Cycle (EUDC). The reason why EUDC is selected is that the velocity changes greatly in it and the time is shorter than other driving cycles, so that the contrast of the performance results with different control strategy is obvious. The global optimal control strategy has a large improvement in terms of fuel economy, SOC variation range is much smaller, and the final SOC is larger compared with the rule-based control strategy, due to the fact that the rule-based control strategy mostly depends on engineering experience and cannot ensure an
TABLE 2: SFC (L/100 km), final SOC (INITIAL 0.7), and eSFC $(\mathrm{L} / 100 \mathrm{~km})$ for a cycle under the EUDC.

\begin{tabular}{|c|c|c|c|}
\hline \multirow{2}{*}{ Strategy } & \multicolumn{3}{|c|}{ Performance } \\
\hline & SFC & eSFC & Final SOC \\
\hline Rule-based & $5.14(100 \%)$ & $4.54(88.3 \%)$ & 0.64 \\
\hline Global optimal & $3.05(59.3 \%)$ & $2.86(55.6 \%)$ & 0.69 \\
\hline Simpler real-time & $3.09(60.1 \%)$ & $2.90(56.4 \%)$ & 0.68 \\
\hline Real-time with ECMS & $3.07(59.7 \%)$ & $2.87(55.8 \%)$ & 0.69 \\
\hline
\end{tabular}

optimal result due to the omission of detailed dynamic models.

From Table 2 and Figure 8, the performances of the simpler real-time control strategy are slightly inferior to that of the global optimal one, in terms of both fuel economy and sustainability of the battery charge. Battery SOC of the global optimal control strategy fluctuates between 0.7 and 0.65 and reaches 0.69 at the end of a cycle. SOC of the simpler real-time control strategy changes in the range of $0.7-0.64$ and attains 0.68 at the end of a cycle. The reason of the results is that the simpler real-time control strategy is local optimization. Nevertheless, the simpler real-time control strategy is more suitable for practical applications because of its short computation time and strong adaptability.

The result of the real-time control strategy with the ECMS is very close to that of the global optimal one in terms of fuel consumption, because the optimization objective of the 


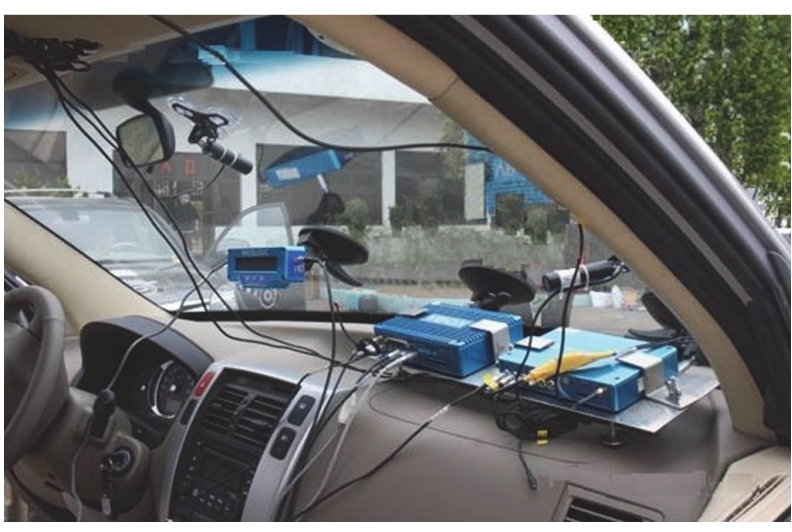

(a) Hardware connection

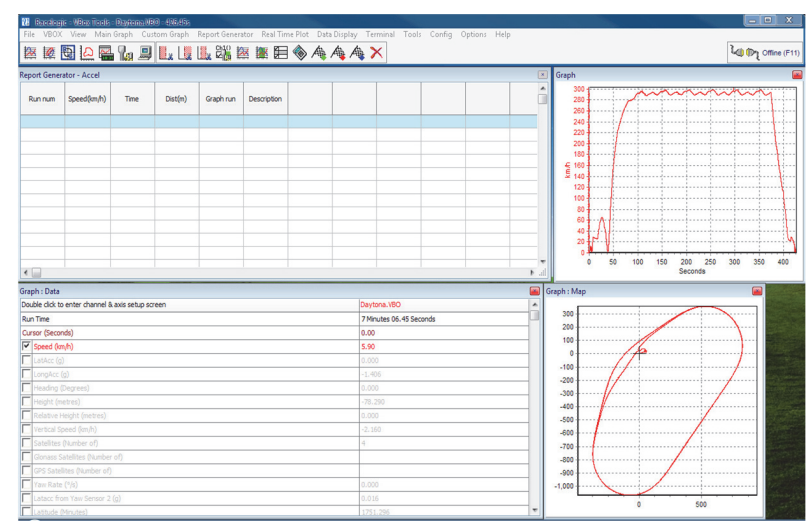

(b) Software interface

FIgURE 9: VBOX3i data acquisition system.

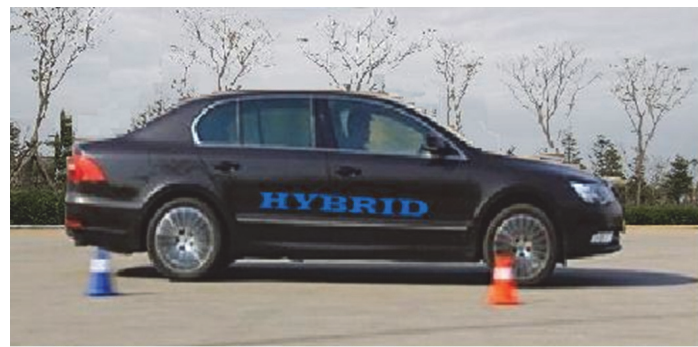

Figure 10: Real vehicles test.

former is the minimum fuel consumption. Although the final SOC of both control strategies is equal, the instantaneous SOC is different during the driving circle. SOC variation of the former is gentle, whereas that of the latter is steep, because the optimization objective of the former takes into account the battery SOC (seen in Section 3), so the former can keep the deviations of SOC at a low level during the whole cycle.

To demonstrate the simulation results further, different driving cycles are selected, such as the Urban Dynamometer Driving Schedule (UDDS), the Highway Fuel Economy Test (HWFET), or the New European Driving Cycle (NEDC). A new variable is introduced for longer running time of the vehicle. When enough repetitions of the cycle are conducted, the SOC tends to a cyclic steady-state. The steady-state specific fuel consumption (ssSFC, liters $/ 100 \mathrm{~km}$ ) is no longer affected by SOC variations and thus represents a good estimation of the equivalent fuel consumption. After five repetitions, the values of ssSFC and eSFC for four control strategies are shown in Table 3 under three driving cycles.

From Table 3, three results can be obtained. Firstly, the ssSFC and eSFC of the same control strategy are different under different driving cycle. According to the value of the ssSFC and eSFC, from small to large, the order is the HWFET, NEDC, and UDDS owing to their different characteristic. The details are as follows. The vehicle velocity always keeps high and varies slightly under the HWFET. The vehicle stops frequently and works in low velocity at most of time under the UDDS. The number of the vehicle stops is moderate; low velocity and high velocity are separated obviously under the NEDC.

Secondly, the ssSFC and eSFC of the real-time control strategy with ECMS are adjacent to those of the global optimal strategy, those of the simpler real-time control strategy are higher than those of these two strategies, and those of the rule-based control strategy are the highest. These results prove that the fuel economy of the real-time control strategy with ECMS can be better under different driving cycle, just conspicuously or inconspicuously.

Thirdly, the value of ssSFC for the same control strategy under the same driving cycle is quite close to the value of eSFC, which confirms the accuracy of the approach used for the evaluation of the equivalent factor.

5.2. Experiments Validation. Aforementioned driving cycles are very useful for long statistical study of the PHEV, for their conditions are easily reproduced later on. Unfortunately, the real driving conditions could not reappear, especially when the traffic condition is very severe where the PHEV suffers frequency start-stop. For this reason, the data acquisition system named VBOX3i based on GPS is used to collect and manage real-world driving cycles. The hardware connection and the software interface of VBOX3i are shown in Figures 9(a) and $9(\mathrm{~b})$, respectively. A lot of the real-world driving cycles are obtained, which can be used as predictive driving cycles.

The characteristics of simulations are easy to be implemented and have lower cost, but they cannot reflect situations comprehensively and actually. Consequently, a large number of tests are performed using real vehicles in actual traffic conditions, shown in Figure 10. According to Figure 5, under the driving cycle shown in Figure 11, test results of parts performance are reported in Figures 12 and 13.

In Figure 11, the predictive driving cycle has subtle difference from the real-time driving cycle, so $k_{\text {neq }}^{\prime}$ need be revised and the nominal equivalent factor $k_{\text {neq }}$ can be acquired. Based on the real-time driving cycle, other parameters are acquired, such as $\mathrm{SOC}_{\text {ref }}$ and $\operatorname{SOC}(t)$. The actual operating points of the ICE and the EM with the real-time with ECMS strategy are different from ones with the others seen from Figures 12 and 


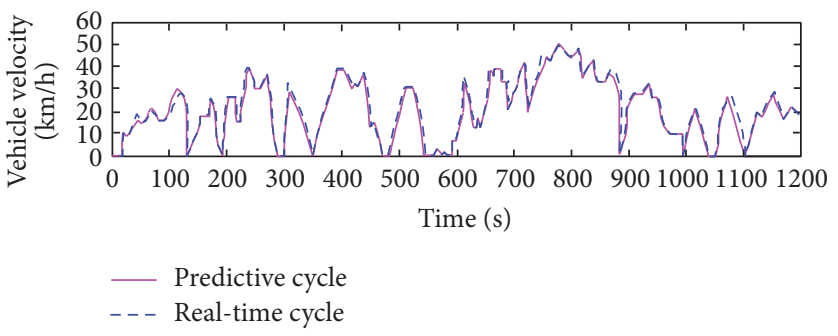

FIGURE 11: Predictive and real-time driving cycle.

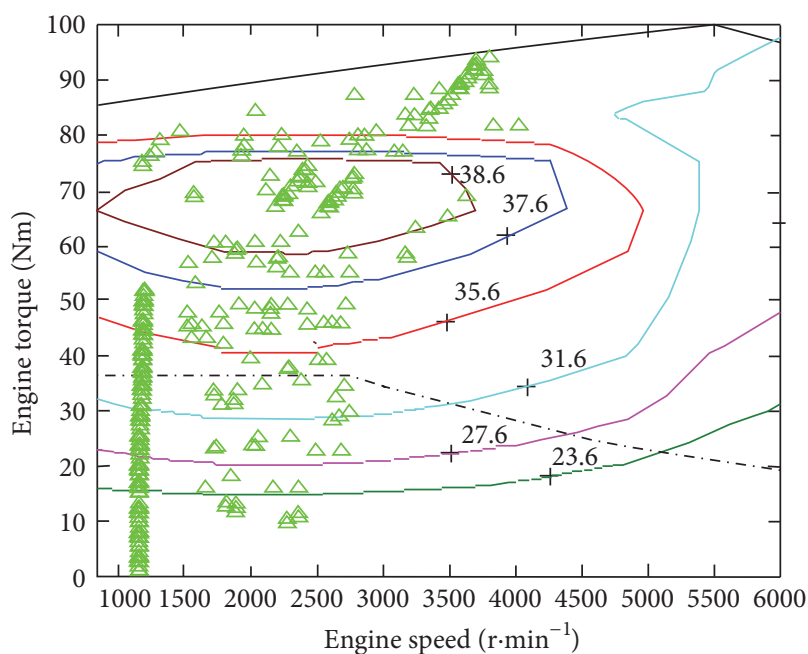

(a) With rule-based

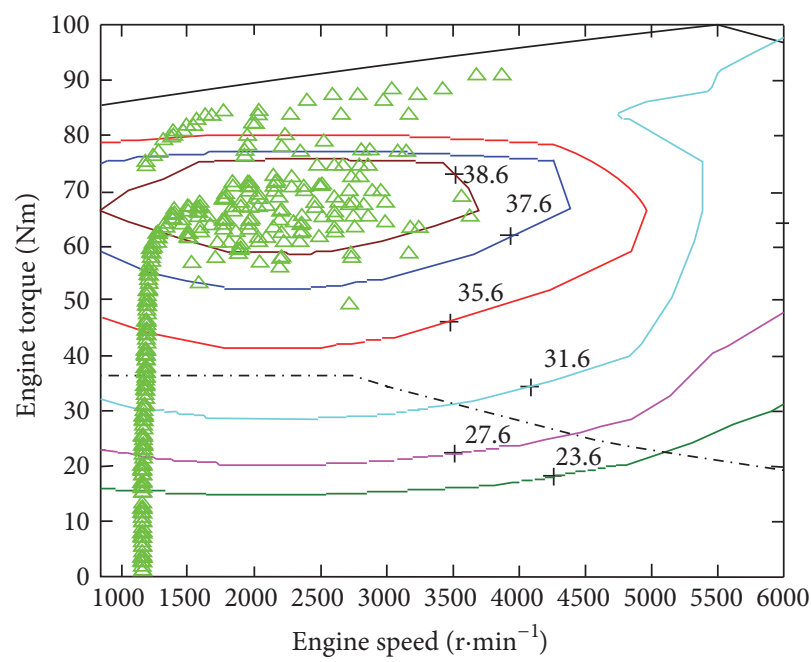

(c) With simpler real-time

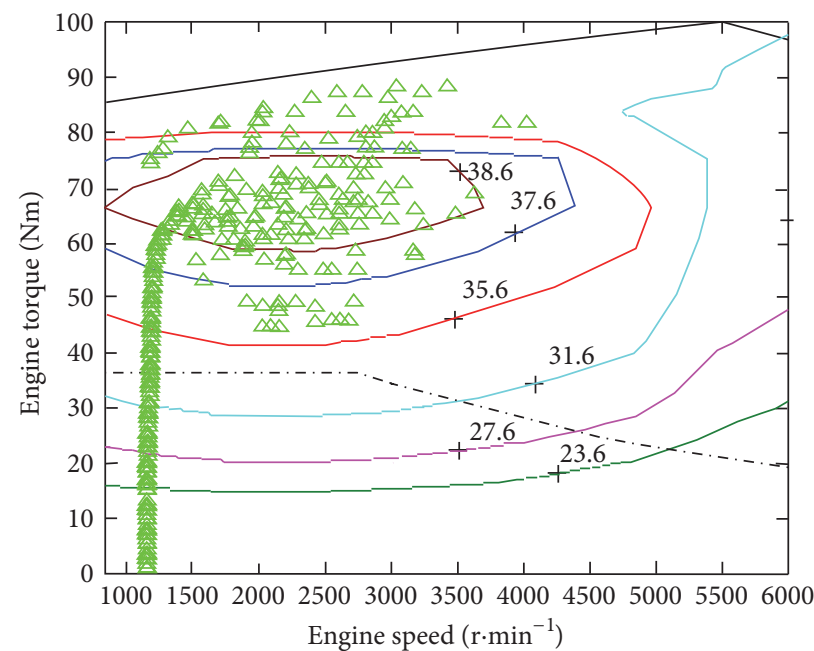

(b) With global optimal

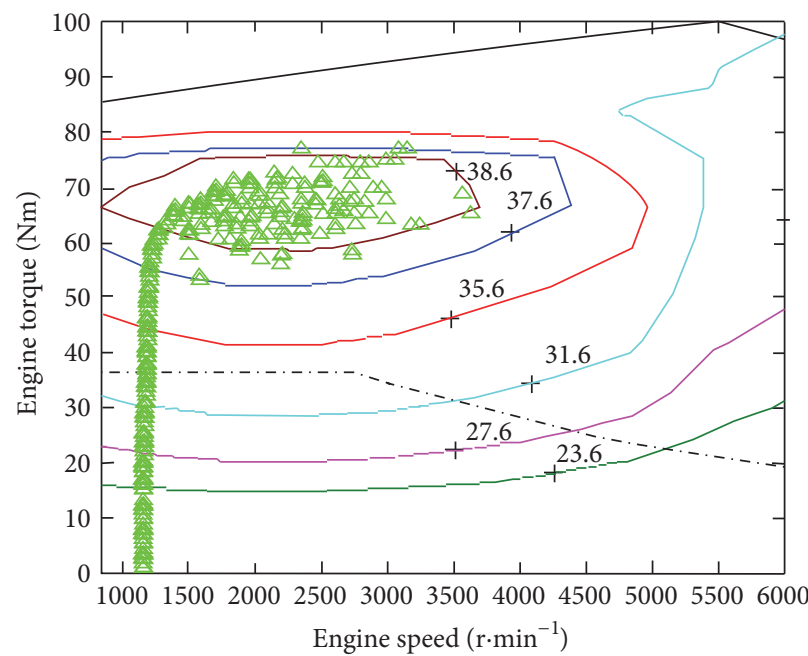

(d) With real-time with ECMS

Figure 12: Operating points of the ICE.

13. The reader should not be misled by the apparent smaller number of disperse operating points. For the real-time with ECMS strategy, the ICE operating points are concentrated in high efficiency region of the ICE with the assistance of the EM, while in the case of global optimal control or simpler real-time control, they are more scattered. They are most scattered in the case of rule-based control. It is also possible to notice that the ICE operating points with the global optimal control are very close to those of the simpler real-time control. These prove that the real-time strategy has best real-time; on the contrary, the global optimal control has no real-time which is not suitable for real vehicle control. Moreover, the control rules of the rule-based control strategy cannot vary promptly according to the real-time driving cycle.

The operating points of the EM are shown in Figure 13. Similarly, both of the motoring and generating efficiency of 


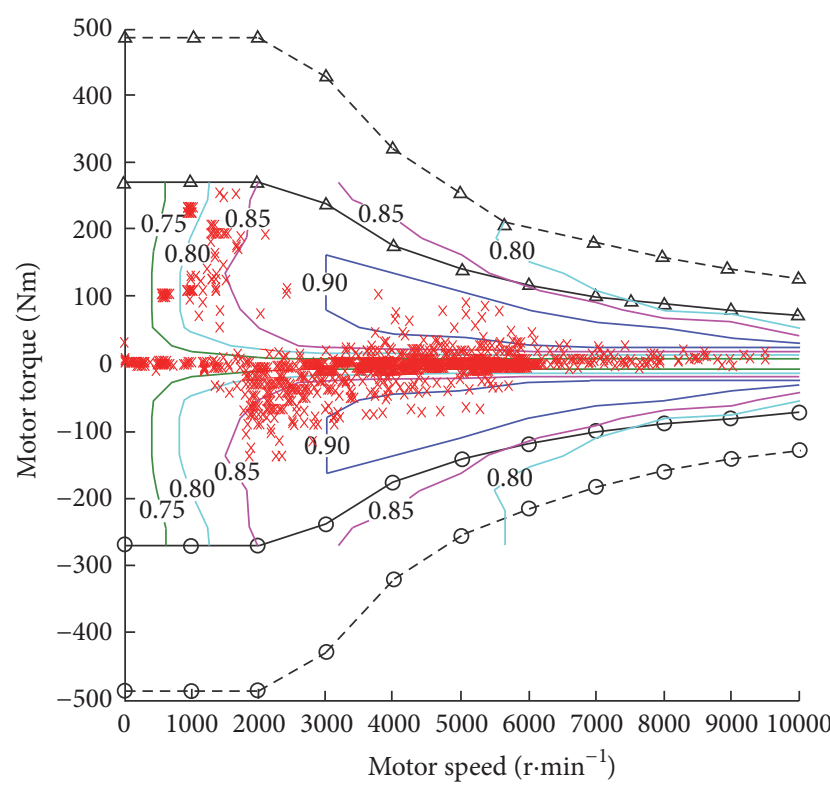
$-\triangle-$ Max motoring torque $\quad-\Theta-$ Max gen. torque
$\triangle$ Max cont. motoring torque $\times$ Actual operating points
- Max cont. gen. torque

(a) With rule-based

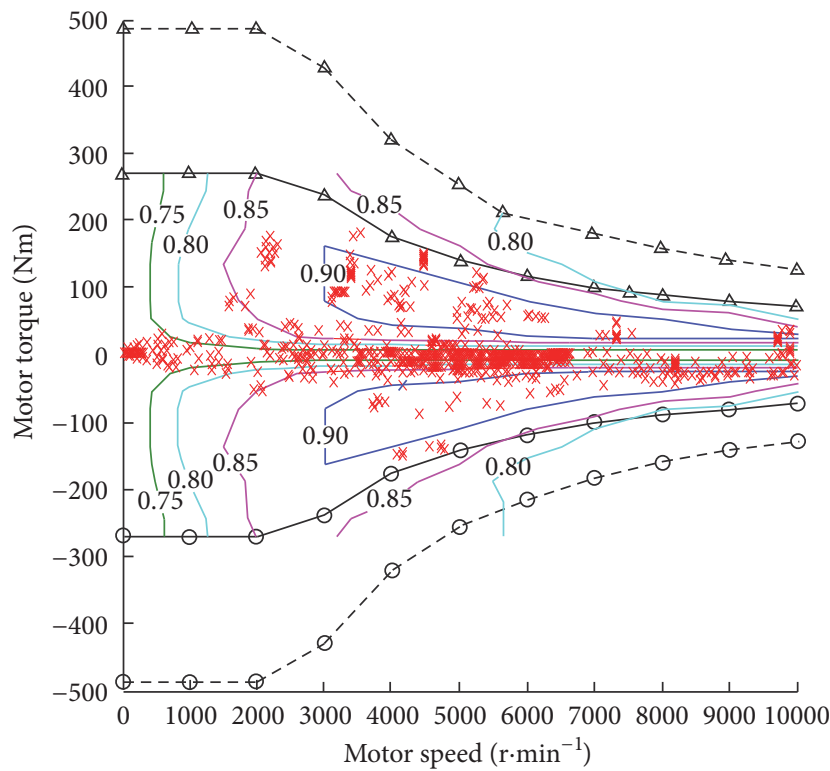

-A- Max motoring torque $\quad-\Theta-$ Max gen. torque

$\triangle$ Max cont. motoring torque $\times$ Actual operating points - Max cont. gen. torque

(c) With simpler real-time

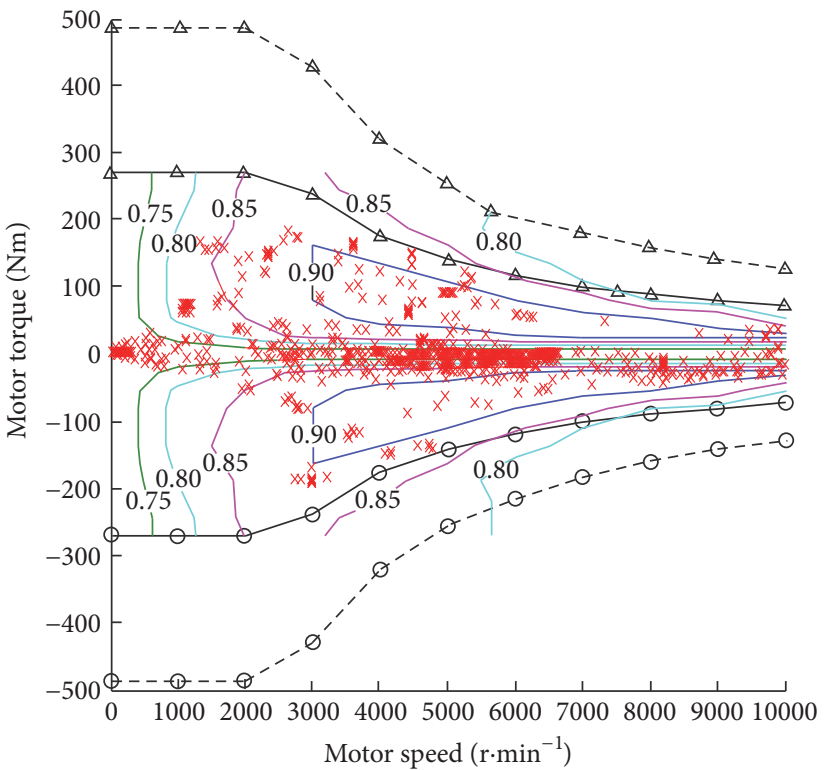

-A- Max motoring torque $\quad-\Theta-$ Max gen. torque

$\triangle$ Max cont. motoring torque $\times$ Actual operating points ○ Max cont. gen. torque

(b) With global optimal

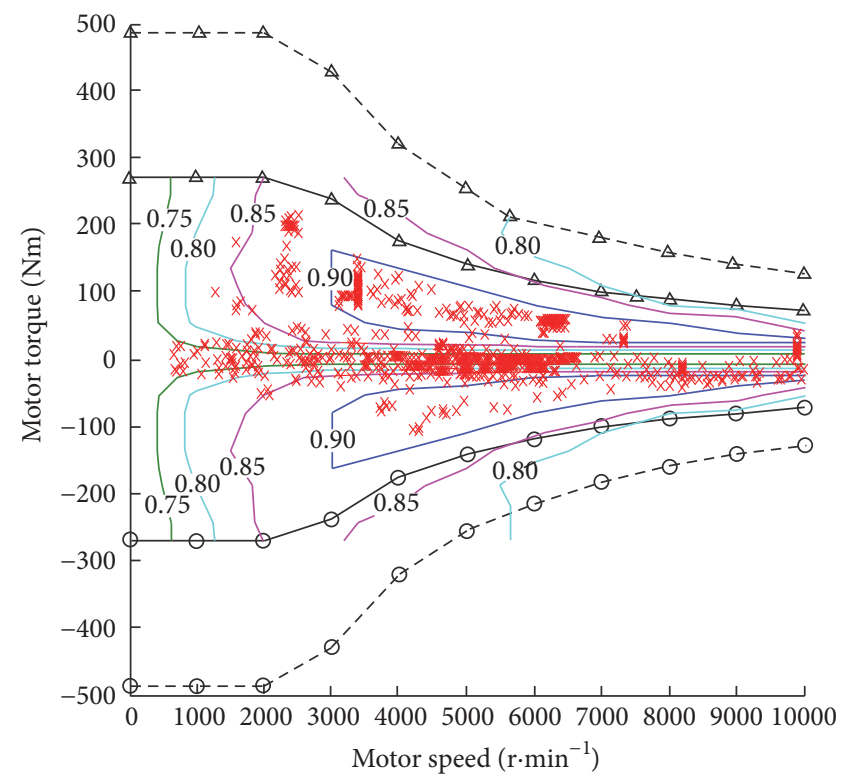

$-\Delta-$ Max motoring torque $\quad-\ominus-$ Max gen. torque
$\triangle$ Max cont. motoring torque $\times$ Actual operating points
$\ominus$ Max cont. gen. torque

(d) With real-time with ECMS

FIGURE 13: Operating points of the EM. 
TABle 3: Comparison between ssSFC (L/100 km) and eSFC $(\mathrm{L} / 100 \mathrm{~km})$ under the UDDS, HWFET, and NEDC.

\begin{tabular}{lcccccc}
\hline Strategy & \multicolumn{3}{c}{ ssSFC } & \multicolumn{4}{c}{ eSFC } \\
& UDDS & HWFET & NEDC & UDDS & HWFET & NEDC \\
\hline $\begin{array}{l}\text { Rule-based } \\
\text { Global }\end{array}$ & 4.85 & 4.25 & 4.81 & 4.82 & 4.23 & 4.80 \\
$\begin{array}{l}\text { optimal } \\
\begin{array}{l}\text { Simpler } \\
\text { real-time }\end{array}\end{array}$ & 2.97 & 2.68 & 2.92 & 2.95 & 2.67 & 2.92 \\
$\begin{array}{l}\text { Real-time } \\
\text { with ECMS }\end{array}$ & 3.17 & 2.79 & 3.17 & 3.18 & 2.78 & 3.16 \\
\hline
\end{tabular}

most of them with the real-time with ECMS strategy are higher than that of the EM with the others. As a result, the vehicle efficiency is increased by using the proposed strategy, which is in accordance with the design purpose.

\section{Conclusions}

The paper has proposed a real-time control strategy with ECMS for a PHEV, whose aim is keeping SOC at a constant reference point with the minimum equivalent fuel consumption. On the basis of the models established of the PHEV, the objective function for the ECMS is evaluated and minimized by selecting a proper value for the torque split control variable. Computation and optimization of the total equivalent fuel consumption are discussed in detail in the paper, in which the approach to evaluating the equivalent factor between fuel energy and electrical energy is new.

The proposed control strategy has been validated by not only computer simulations but also real vehicle tests compared with other three control strategies under a large database of driving cycles, including standard cycles (the EUDC, UDDS, HWFET, NEDC, and so on), actual vehicle velocity profiles extracted from fleet studies data, and realtime driving cycles. The implementation of the proposed control strategy is divided into two steps. Firstly, the predictive models of the driving cycle are selected based on the historical traffic information. Secondly, the parameters are revised according to the real-time driving cycle. As a result, great improvement is achieved in computational efficiency and accuracy.

The following results have been illustrated.

(1) The approach used for the evaluation of the equivalent factor is correct.

(2) Equivalent fuel economy of the proposed control strategy is suboptimal, next to that of the global optimal control strategy.

(3) The proposed control strategy can always keep the deviations of SOC at a low level.

(4) The parts performances with the proposed control strategy are better than that with other three control strategies. The proposed control strategy and method can be also applied to the generally valid ECMS.

\section{Competing Interests}

The authors declare that they have no competing interests.

\section{Acknowledgments}

This work was partially supported by National Natural Science Foundation of China (Grants nos. 61473115, 51375145, and U1404610) and Science and Technology Innovative Foundation for Distinguished Young Scholar of Henan Province (Grant no. 144100510004).

\section{References}

[1] C. C. Chan, "Outlook of electric, hybrid and fuel cell vehicles," Journal of Automotive Safety and Energy, vol. 2, no. 1, pp. 12-24, 2011.

[2] H. Borhan, A. Vahidi, A. M. Phillips et al., "MPC-based energy management of a power-split hybrid electric vehicle," IEEE Transactions on Control Systems Technology, vol. 20, no. 3, pp. 593-603, 2012.

[3] F. R. Salmasi, "Control strategies for hybrid electric vehicles: evolution, classification, comparison, and future trends," IEEE Transactions on Vehicular Technology, vol. 56, no. 5 I, pp. 23932404, 2007.

[4] K. Van Berkel, T. Hofman, B. Vroemen, and M. Steinbuch, "Optimal control of a mechanical hybrid powertrain," IEEE Transactions on Vehicular Technology, vol. 61, no. 2, pp. 485-497, 2012.

[5] S. G. Li, S. M. Sharkh, F. C. Walsh, and C. N. Zhang, "Energy and battery management of a plug-in series hybrid electric vehicle using fuzzy logic," IEEE Transactions on Vehicular Technology, vol. 60, no. 8, pp. 3571-3585, 2011.

[6] S. J. Moura, H. K. Fathy, D. S. Callaway, and J. L. Stein, "A stochastic optimal control approach for power management in plug-in hybrid electric vehicles," IEEE Transactions on Control Systems Technology, vol. 19, no. 3, pp. 545-555, 2011.

[7] Q. M. Gong, Y. Y. Li, and Z.-R. Peng, "Trip-based optimal power management of plug-in hybrid electric vehicles," IEEE Transactions on Vehicular Technology, vol. 57, no. 6, pp. 3393-3401, 2008.

[8] Z. Chen, C. C. Mi, J. Xu et al., "Energy management for a power-split plug-in hybrid electric vehicle based on dynamic programming and neural networks," IEEE Transaction on Vehicular Technology, vol. 63, pp. 1567-1580, 2014.

[9] H. Lee, S. Cha, H. Kim et al., "Energy management strategy of hybrid electric vehicle using stochastic dynamic programming," SAE Paper 2015-01-0019, 2015.

[10] F. Roy, F. Ossart, and C. Marchand, "An optimal energetic approach for systemic design of hybrid powertrain," in Proceedings of the IEEE Vehicle Power and Propulsion Conference (VPPC '14), IEEE, Coimbra, Portugal, October 2014.

[11] D. Karbowski, A. Rousseau, S. Pagerit et al., "Plug-in vehicle control strategy: from global optimization to real-time application," in Proceedings of the 22nd Electric Vehicle Symposium, pp. 24-27, Yokohama, Japan, October 2006.

[12] S. G. Wirasingha and A. Emadi, "Classification and review of control strategies for plug-in hybrid electric vehicles," IEEE Transactions on Vehicular Technology, vol. 60, no. 1, pp. 111-122, 2011.

[13] L. Wang, E. G. Collins Jr., and H. Li, "Optimal design and realtime control for energy management in electric vehicles," IEEE 
Transactions on Vehicular Technology, vol. 60, no. 4, pp. 14191429, 2011.

[14] R. Razavian, N. L. Azad, and J. McPhee, "On real-time optimal control of a series Hybrid Electric Vehicle with an ultra-capacitor," in Proceedings of the American Control Conference (ACC '12), pp. 547-552, Montreal, Canada, June 2012.

[15] N. Kim, S. Cha, and H. Peng, "Optimal control of hybrid electric vehicles based on Pontryagin's minimum principle," IEEE Transactions on Control Systems Technology, vol. 19, no. 5, pp. 1279-1287, 2011.

[16] G. Paganelli, S. Delprat, T. Guerra et al., "Equivalent consumption minimization strategy for parallel hybrid powertrains," in Proceedings of the IEEE 55th Vehicle Technology Conference, pp. 2076-2081, Birmingham, Ala, USA, May 2002.

[17] A. Sciarretta, M. Back, and L. Guzzella, "Optimal control of parallel hybrid electric vehicles," IEEE Transactions on Control Systems Technology, vol. 12, no. 3, pp. 352-363, 2004.

[18] V. Sezer, M. Gokasan, and S. Bogosyan, "A novel ECMS and combined cost map approach for high-efficiency series hybrid electric vehicles," IEEE Transactions on Vehicular Technology, vol. 60, no. 8, pp. 3557-3570, 2011.

[19] N. Kim, S. W. Cha, and H. Peng, "Optimal equivalent fuel consumption for hybrid electric vehicles," IEEE Transactions on Control Systems Technology, vol. 20, no. 3, pp. 817-825, 2012.

[20] S. Adhikari, S. K. Halgamuge, and H. C. Watson, "An online power-balancing strategy for a parallel hybrid electric vehicle assisted by an integrated starter generator," IEEE Transactions on Vehicular Technology, vol. 59, no. 6, pp. 2689-2699, 2010.

[21] W. Zhou, Y. Gao, D. Tong, C. Ji, and J. Fang, "Adaptive exponential synchronization in pth moment of neutral-type neural networks with time delays and Markovian switching," International Journal of Control, Automation and Systems, vol. 11, no. 4, pp. 845-851, 2013. 


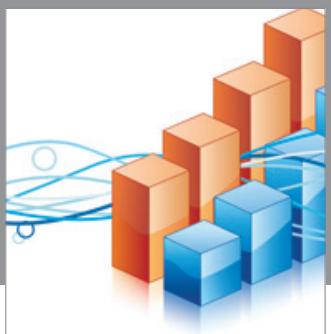

Advances in

Operations Research

vatem alat4

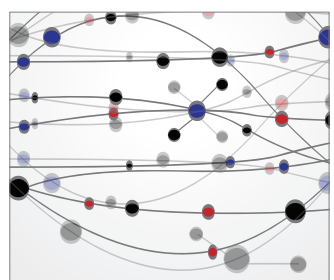

\section{The Scientific} World Journal
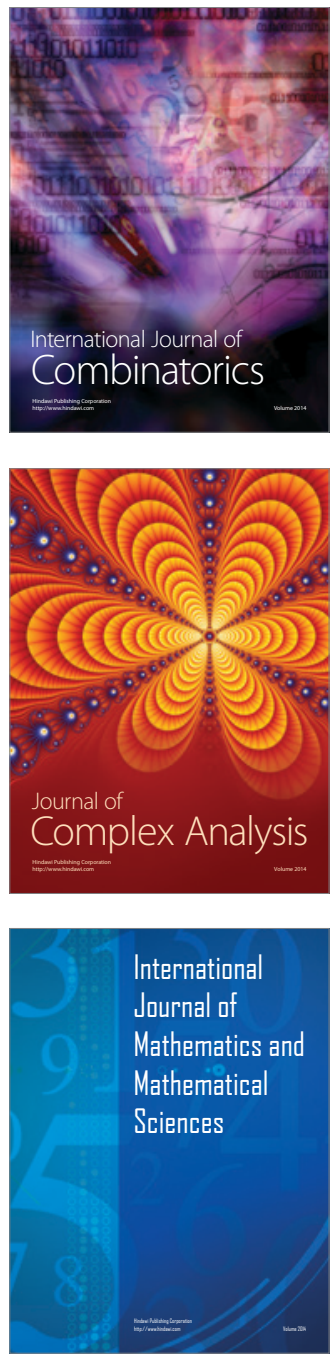
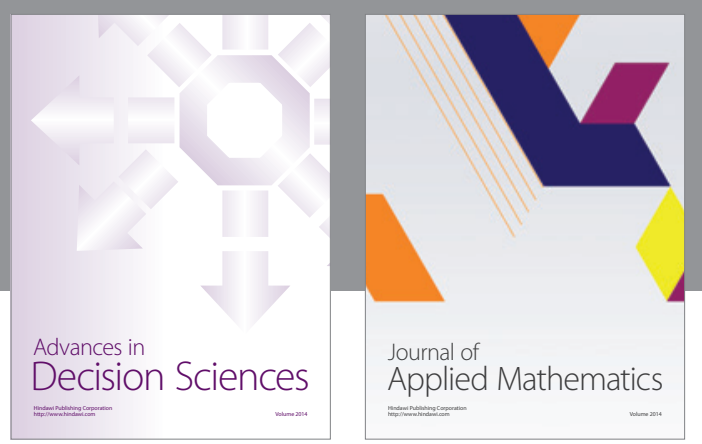

Algebra

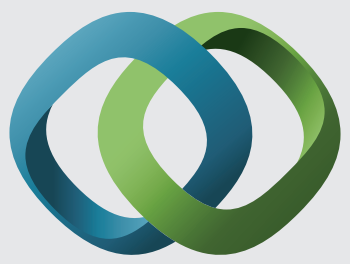

\section{Hindawi}

Submit your manuscripts at

https://www.hindawi.com
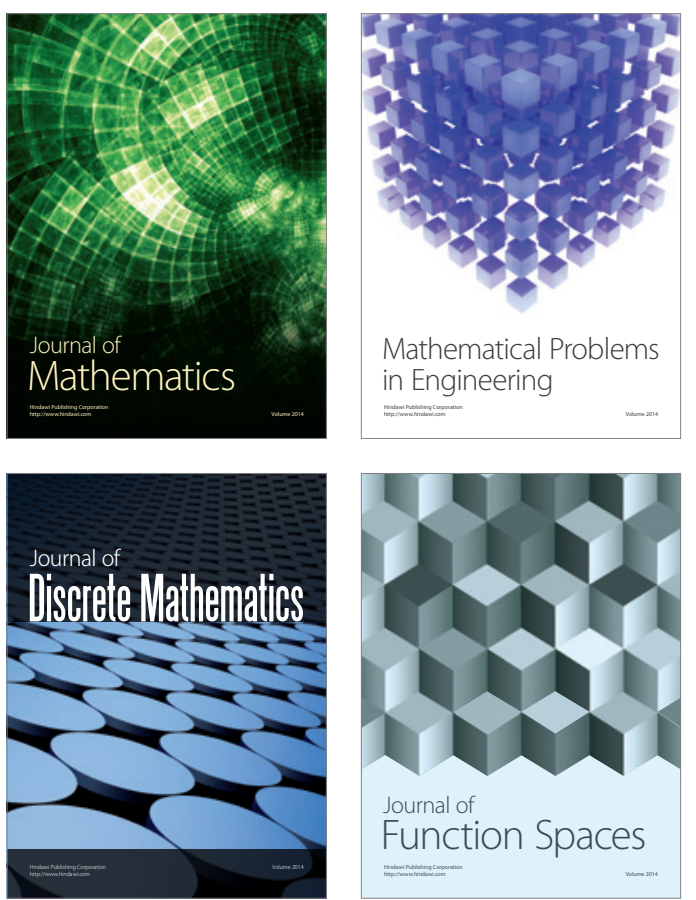

Mathematical Problems in Engineering
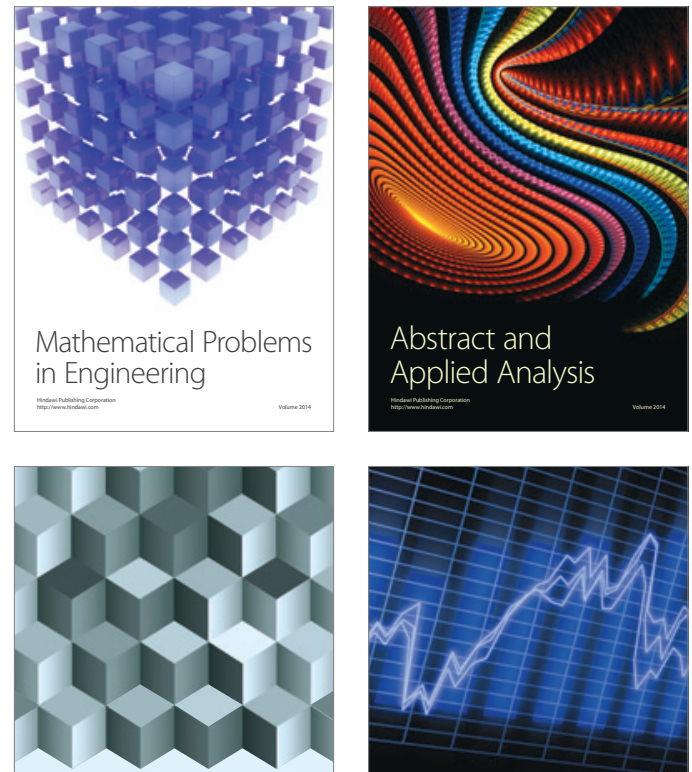

Journal of

Function Spaces

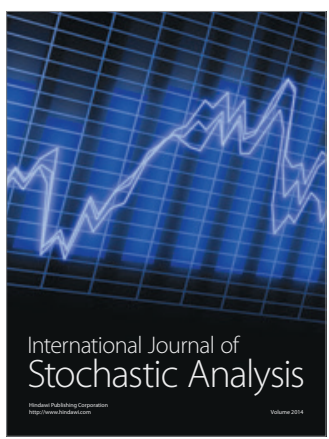

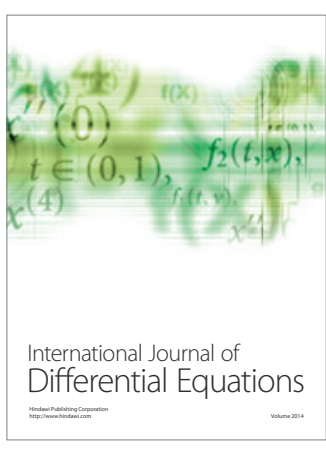
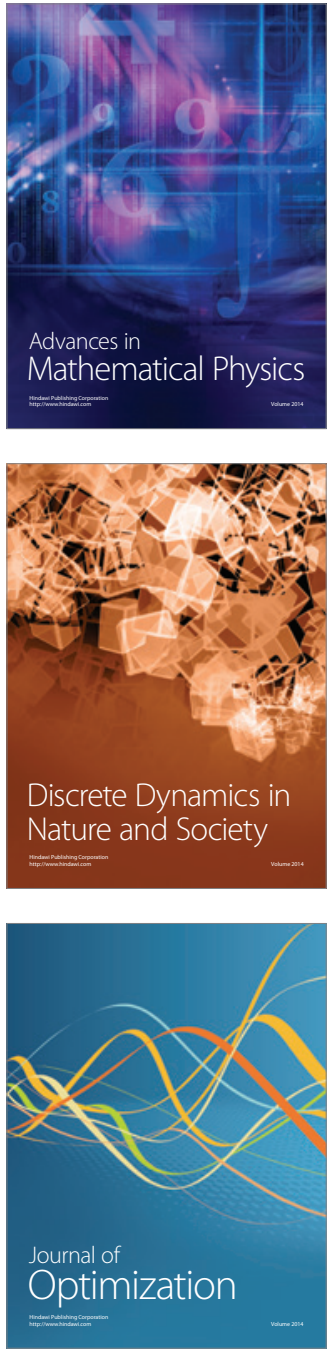